\title{
Adaptation of the Young Adults' Cigarette Dependence (YACD) Scale for the development and validation of the Adolescent Cigarette Dependence Scale (ACDS)
}

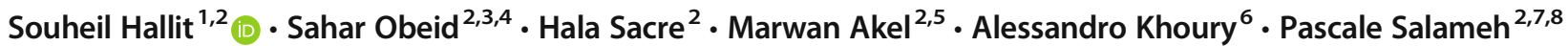

Received: 28 October 2020 / Accepted: 21 January 2021 / Published online: 4 February 2021

(C) The Author(s), under exclusive licence to Springer-Verlag GmbH, DE part of Springer Nature 2021

\begin{abstract}
Vulnerable adolescents may lose control over cigarette use within a day or two after smoking their first cigarette, as nicotine dependence (ND) symptoms may appear before actual daily smoking and usually occur at low levels of daily smoking. Moreover, smoking can cause illnesses and promote other types of substance abuse. Therefore, it was deemed essential to adapt the Young Adults' Cigarette Dependence (YACD) Scale to develop and validate the Adolescent Cigarette Dependence Scale (ACDS) in Lebanese adolescents and evaluate whether cumulative cigarette smoking is associated with nicotine dependence and symptoms of dependence. A cross-sectional study was carried out between August and September 2020, during the partial sanitary lockdown imposed by the government to limit COVID-19, coinciding with the summer holidays for most Lebanese. The total sample consisted of 564 community-dwelling adolescents aged 13 to 18 . A factor analysis was performed using a varimax rotation. The Kaiser-Meyer-Olkin (KMO) measurement of sampling adequacy and Bartlett's sphericity test were appropriate. The factors retained corresponded to Eigenvalues $>1$. Cronbach's alpha value represented the internal consistency of the scale. The Hooked on Nicotine Checklist (HONC) was used to screen for dependence symptoms. All items of the YACD were extracted, except for items 4 (smoking to concentrate at work) and 18 (smoking as a habit), which yielded a three-factor solution (factor 1 = smoking patterns; factor $2=$ positive and negative reinforcements; factor $3=$ smoking cessation) with Eigenvalues $>1$ (variance explained $=56.18 \% ; \mathrm{KMO}=0.784 ;$ Bartlett's sphericity test $p<0.001 ; \alpha_{\text {Cronbach }}=0.875$ ). The newly generated scale was termed Adolescent Cigarette Dependence Scale (ACDS) and consisted of 19 items. Higher ACDS scores (higher cigarette dependence) were significantly associated with higher HONC scores (higher symptoms of dependence) (rho $=0.647 ; p<0.001$ ). Having deceased $(B=8.54)$ or divorced $(B=4.26)$ parents and higher cumulative cigarette smoking $(B=0.29)$ were significantly associated with higher ACDS scores. Higher cumulative cigarette smoking $(B=0.05)$ was significantly associated with higher HONC scores due to cigarettes. This study could validate a new tool to evaluate nicotine dependence among Lebanese adolescents, the Adolescent Cigarette Dependence Scale (ACDS). This version adapted from the Young Adults' Cigarette Dependence (YACD) Scale is reliable and valuable and correlates well with other scales, such as the HONC. However, further studies are necessary to improve this instrument, adding new questions useful for the diagnosis and evaluation of cigarette dependence.
\end{abstract}

Keywords Cigarette $\cdot$ Dependence $\cdot$ Adolescents $\cdot$ YACD $\cdot$ Symptoms

Responsible Editor: Lotfi Aleya

Souheil Hallit

souheilhallit@hotmail.com

Pascale Salameh

pascalesalameh1@hotmail.com

1 Faculty of Medicine and Medical Sciences, Holy Spirit University of Kaslik (USEK), Jounieh, Lebanon

2 INSPECT-LB: Institut National de Santé Publique, Épidémiologie Clinique et Toxicologie-Liban, Beirut, Lebanon
3 Faculty of Arts and Sciences, Holy Spirit University of Kaslik (USEK), Jounieh, Lebanon

4 Research and Psychology Departments, Psychiatric Hospital of the Cross, Jal Eddib, Lebanon

5 School of Pharmacy, Lebanese International University, Beirut, Lebanon

6 Faculty of Medicine, American University of Beirut, Beirut, Lebanon

7 Faculty of Pharmacy, Lebanese University, Beirut, Lebanon

8 Faculty of Medicine, University of Nicosia, Nicosia, Cyprus 


\section{Introduction}

Adolescence is a critical period in life at the psychological, social, and cognitive levels, with higher susceptibility to substance use and other risky behaviors (Dir et al. 2017; Hammerslag and Gulley 2016), such as smoking, which remains one of the most health-threatening habits. According to the World Health Organization (WHO), among adolescents aged 13 to 15 , one in five boys and one in ten girls smoke tobacco (World Health Organization Behaviours 2020). Moreover, in 12 of the 158 studied countries, 1 in 3 younger adolescents uses cigarettes, with statistics showing that smoking is at the highest level in Europe and the Western Pacific (World Health Organization Behaviours 2020). In Lebanon, rates are becoming alarming; a previous Lebanese study found that the weighted smoking prevalence of frequent adolescent smokers was 5.1\% (Bejjani et al. 2012). Tobacco use usually begins in adolescence, between the ages of 10 and 13 , and increases more rapidly between the ages of 14 and 15 (Bejjani et al. 2012).

Nicotine dependence (ND) is characterized by tolerance, craving, withdrawal symptoms in case of abstinence, loss of control over the number of cigarettes and the time spent smoking, unsuccessful attempts to quit, neglect of important activities, and smoking despite physical or psychological problems (Griesler et al. 2016). Withdrawal symptoms due to nicotine include but are not limited to depressed mood, irritability, nervousness, anger, anxiety, trouble focusing, and impatience (Griesler et al. 2016). The unpublished results of a recent national study conducted among adolescents revealed that the prevalence of moderate to high cigarette dependence was $21.8 \%$. Additionally, long smoking sessions and early initiation of smoking are strongly associated with more ND (Bahelah et al. 2016).

Several instruments were developed to assess nicotine dependence. One of the oldest and most commonly used is the Fagerström Test for Nicotine Dependence (FTND) (Fagerstrom 1978; Heatherton et al. 1991), but it remains a suboptimal tool since it explores one dimension of ND (Shiffman et al. 2004). In 2004, the Nicotine Dependence Syndrome Scale (NDSS) was developed to assess ND in adults, taking into consideration the multiple dimensions of ND (Shiffman et al. 2004), and validated in 2005 among adolescents (Clark et al. 2005). Another tool used to assess ND is the Hooked on Nicotine Checklist (HONC) (DiFranza et al. 2000) created after a theory-based explanation of ND in adolescents, hypothesizing that individuals are hooked when they have experienced a loss of autonomy over their use of nicotine (DiFranza et al. 2000). At the Lebanese level, the Young Adults' Cigarette Dependence (YACD) scale has been validated among university students (Salameh et al. 2013), relying on the FTND scale, Diagnostic and Statistical Manual of
Mental Disorders (DSM-IV), and International Classification of Diseases (ICD-10) dependence components. However, no scales have been validated among adolescents in Lebanon.

Vulnerable adolescents may lose control over cigarette use within a day or two after smoking their first cigarette, as ND symptoms may appear before actual daily smoking and usually occur at low levels of daily smoking (DiFranza et al. 2007). Moreover, smoking can cause illnesses and promote other types of substance abuse. Therefore, it was deemed essential to adapt the Young Adults' Cigarette Dependence (YACD) Scale to develop and validate the Adolescent Cigarette Dependence Scale (ACDS) in Lebanese adolescents and evaluate whether cumulative cigarette smoking is associated with nicotine dependence and symptoms of dependence.

\section{Methods}

\section{General study design}

A cross-sectional study was carried out between August and September 2020, during the partial sanitary lockdown imposed by the government to limit COVID-19, coinciding with the summer holidays for most Lebanese. The sample consisted of community-dwelling adolescents aged 13 to 18 . Due to the restrictions on gatherings and the non-practical and risky side of face-to-face interviews, an online survey was created on Google forms (https://bit.ly/2HzlyD8), and the link shared with participants from all districts/governorates of Lebanon (Beirut, Mount Lebanon, North Lebanon, South Lebanon, and Beqaa), using the snowball sampling technique; the participants were asked to fill the survey online and share the link with other adolescents, smokers, and non-smokers. All participants aged 13 to 18 were eligible. The total sample comprised 564 adolescents.

\section{Sample size calculation}

The adequate sample size was determined according to the ratio of 1:10 (ten observations per variable) suggested by Comrey and Lee (2013). The minimum needed sample was 210 participants, as the YACD scale consisted of 21 items.

\section{Questionnaire and variables}

A self-administered anonymous questionnaire with closedended questions was shared online with participants. It was in Arabic, the native language in Lebanon, and required approximately $10 \mathrm{~min}$ to complete. The questionnaire consisted of two sections. The first part clarified sociodemographic characteristics, including age, gender, area of residence, educational level, and data to determine the household crowding 
index (HCI), reflecting the socioeconomic status (SES). The latter is calculated by dividing the number of persons living in the house by the number of rooms (excluding bathrooms and kitchen); the higher the HCI, the lower the SES. The second part gathered information about the smoking status, such as the time spent looking for cigarettes, the number of cigarettes, the age of initiation, the importance of smoking, money spent on smoking. It also included the following scales:

\section{Young Adults' Cigarette Dependence (YACD) scale}

This 21-item scale is used to assess cigarette smoking dependence (Salameh et al. 2013). Answers are rated on a 4-point Likert scale. The total score is calculated by summing all responses, with higher scores reflecting more ND. In this study, Cronbach's alpha was 0.886 .

\section{Hooked on Nicotine Checklist}

This tool used an indicator of reduced autonomy that includes 10 items. A score of zero by answering No to all ten questions means that the subject has full control over the use of tobacco. Higher scores indicate higher nicotine dependence symptoms (DiFranza et al. 2002). In this study, Cronbach's alpha was 0.892 .

\section{Statistical analysis}

Statistical analysis was done on SPSS software version 23. Data were weighted to the general population according to age, gender, and governorate. A factor analysis was performed using the principal component analysis technique to confirm the legitimacy of the construct of the YACD scale in our sample; a varimax rotation was selected since the extracted factors were not highly correlated. The Kaiser-Meyer-Olkin (KMO) measurement of sampling adequacy and Bartlett's sphericity test were appropriate. The factors retained corresponded to Eigenvalues greater than one. Cronbach's alpha value represented the internal consistency of the scale. The YACD and HONC scores were not normally distributed; thus, non-parametric tests were applied; the Mann-Whitney test checked for an association between the dependent variables (YACD and HONC) and dichotomous variables, while the Kruskal-Wallis test compared the means of three or more groups. Spearman correlation was used to compare two continuous variables. Forward linear regressions were carried out to assess factors associated with the YACD and HONC scores, taking into account potential confounding variables that showed a $p<0.2$ in the bivariate analysis as independent variables. Assumptions of linear regressions were checked before accepting the final results. Finally, a multivariate analysis of covariance (MANCOVA) was carried out to assess sociodemographic and family-related variables associated with each factor obtained in the factor analysis, taken as dependent variables. A value of $P<0.05$ was considered significant.

\section{Results}

The mean age of the participants was $16.37 \pm 1.65$ years, $50.7 \%$ were males, and $35.8 \%$ were exclusive cigarette smokers. Table 1 summarizes other sociodemographic characteristics. The mean number of cigarettes smoked per day was 3.00 (SD: 6.14), and the mean number of years of cigarette smoking was 0.59 (SD: 1.29). Cumulative cigarette smoking was 5.90 (SD: 17.33).

\section{Factor analysis}

Sample $1(n=400)$ was used for the factor analysis; all items of the YACD were extracted, except for items 4 and 18, which yielded a three-factor solution (factor $1=$ smoking patterns; factor 2 = positive and negative reinforcements; factor $3=$ smoking cessation) with Eigenvalues $>1$ (variance explained

Table 1 Sociodemographic characteristics of the participants $(N=564)$

\begin{tabular}{|c|c|c|}
\hline & Frequency & Percentage $(\%)$ \\
\hline \multicolumn{3}{|l|}{ Gender } \\
\hline Male & 286 & $50.7 \%$ \\
\hline Female & 278 & $49.3 \%$ \\
\hline \multicolumn{3}{|l|}{ Education level } \\
\hline Complementary & 148 & $26.2 \%$ \\
\hline Secondary & 416 & $73.8 \%$ \\
\hline \multicolumn{3}{|l|}{ Mouhafaza } \\
\hline Beirut & 207 & $36.7 \%$ \\
\hline Mont Lebanon & 202 & $35.8 \%$ \\
\hline North & 42 & $7.4 \%$ \\
\hline South & 73 & $12.9 \%$ \\
\hline Beqaa & 40 & $7.1 \%$ \\
\hline \multicolumn{3}{|l|}{ Parents' status } \\
\hline Living together & 466 & $82.6 \%$ \\
\hline Divorced & 50 & $8.9 \%$ \\
\hline One of the parents is deceased & 48 & $8.5 \%$ \\
\hline \multicolumn{3}{|l|}{ Current cigarette smoking } \\
\hline No & 362 & $64.2 \%$ \\
\hline \multirow[t]{2}{*}{ Yes } & 202 & $35.8 \%$ \\
\hline & Mean & SD \\
\hline Age (in years) & 16.37 & 1.65 \\
\hline Household crowding index & 1.07 & 0.41 \\
\hline Number of cigarettes smoked per day & 3.00 & 6.14 \\
\hline Duration of cigarette smoking (in years) & 0.59 & 1.29 \\
\hline YACD score & 22.04 & 11.45 \\
\hline HONC score & 5.38 & 3.35 \\
\hline
\end{tabular}


$=56.18 \% ; \mathrm{KMO}=0.784 ;$ Bartlett's sphericity test $p<0.001$; $\left.\alpha_{\text {Cronbach }}=0.875\right)($ Table 2$)$. The newly generated scale was termed Adolescent Cigarette Dependence Scale (ACDS) and consisted of 19 items.

\section{Bivariate analysis}

Higher ACDS scores were found in males compared with females, in those who have a deceased parent, and in those living in Beqaa compared with the other governorates. Moreover, higher ACDS scores were associated with higher cumulative cigarette smoking.

Higher HONC scores due to cigarettes were found in males compared with females, in those who have a deceased parent, in those with a complementary level of education, and those living in Beqaa compared with the other governorates. Furthermore, higher HONC scores due to cigarettes were associated with higher cumulative cigarette smoking (Table 3).

Finally, higher ACDS scores (higher cigarette dependence) were significantly associated with higher HONC scores (higher symptoms of dependence) (rho $=0.647 ; p<0.001$ ).

\section{Multivariable analysis}

The results of a first linear regression, taking the ACDS score as the dependent variable, showed that having a deceased $(B=$
8.54) or divorced ( $B=4.26)$ parents and higher cumulative cigarette smoking $(B=0.29)$ were significantly associated with higher ACDS scores (Table 4, model 1).

The results of a second linear regression, taking the HONC score as the dependent variable, showed that higher cumulative cigarette smoking $(B=0.05)$ was significantly associated with higher HONC scores due to cigarettes (Table 4, model 2).

Adolescents who had a deceased parent had higher smoking patterns (higher factor 1 scores) and higher positive and negative reinforcements (higher factor 2 scores) compared with those who had parents living together (Table 5, models 1 and 2). Higher household crowding index (reflecting a lower socioeconomic status) and having a deceased parent or divorced parents compared with living together were significantly associated with more smoking cessation (higher factor 3 scores) (Table 5, model 3).

\section{Discussion}

Our results demonstrated that the newly generated Adolescent Cigarette Dependence Scale is an adequate tool to evaluate nicotine dependence among Lebanese adolescents. Furthermore, having deceased or divorced parents was

Table 2 Factor analysis of the YACD scale items using the varimax rotation

\begin{tabular}{|c|c|c|c|c|}
\hline Variable & Item number & Factor 1 & Factor 2 & Factor 3 \\
\hline Smoking to decrease nervousness & 10 & 0.806 & & \\
\hline How much money, in proportion to income, are you ready to pay for cigarette? & 19 & 0.747 & & \\
\hline How soon after you wake up do you smoke your first cigarette? & 5 & 0.715 & & \\
\hline Smoking to increase morale & 7 & 0.697 & & \\
\hline How many cigarettes/day do you smoke? & 2 & 0.660 & & \\
\hline Are you ready not to eat for a cigarette? & 21 & 0.648 & & \\
\hline Smoking to avoid eating & 13 & 0.575 & & \\
\hline Smoking cigarette even when alone & 14 & 0.541 & & \\
\hline Do you smoke more frequently in the morning than the rest of the day? & 3 & 0.490 & & \\
\hline Smoking for pleasure & 8 & & 0.820 & \\
\hline Smoking to concentrate while working & 9 & & 0.808 & \\
\hline Do you prefer smoking cigarettes over going to the theater or other activities? & 16 & & 0.787 & \\
\hline Do you find it difficult to refrain from smoking where it is forbidden? & 1 & & 0.718 & \\
\hline Are you ready to leave your family on a holiday to go and search for cigarettes? & 15 & & 0.653 & \\
\hline Smoking by habit & 12 & & 0.548 & \\
\hline Smoking for conviviality & 11 & & & 0.817 \\
\hline Number of days you could stop smoking cigarettes & 17 & & & 0.718 \\
\hline How many times were you able to stop smoking for more than a week? & 20 & & & 0.617 \\
\hline Which cigarette would you mostly hate to give up? & 6 & & & 0.586 \\
\hline Percentage of variance explained & & 36.15 & 10.82 & 9.21 \\
\hline Cronbach's alpha & & 0.857 & 0.851 & 0.246 \\
\hline
\end{tabular}


Table 3 Bivariate analysis of factors associated with the cigarette dependence (ACDS) score and symptoms of dependence (HONC scores) due to cigarette smoking

\begin{tabular}{|c|c|c|}
\hline Variable & ACDS score & HONC cigarette \\
\hline \multicolumn{3}{|l|}{ Gender } \\
\hline Male & $22.64 \pm 12.35$ & $5.66 \pm 3.39$ \\
\hline Female & $17.90 \pm 10.41$ & $4.28 \pm 3.27$ \\
\hline$p$ & 0.001 & $<0.001$ \\
\hline \multicolumn{3}{|l|}{ Parents' status } \\
\hline Living together & $18.45 \pm 10.16$ & $5.08 \pm 3.60$ \\
\hline Divorced & $22.24 \pm 9.60$ & $4.49 \pm 3.37$ \\
\hline One parent is deceased & $32.26 \pm 14.46$ & $6.42 \pm 2.00$ \\
\hline$p$ & $<0.004$ & 0.041 \\
\hline \multicolumn{3}{|l|}{ Education level } \\
\hline Complementary & $22.35 \pm 14.08$ & $5.73 \pm 3.50$ \\
\hline Secondary & $20.39 \pm 10.42$ & $4.89 \pm 3.31$ \\
\hline$p$ & 0.817 & 0.026 \\
\hline \multicolumn{3}{|l|}{ Governorate } \\
\hline Beirut & $22.44 \pm 10.89$ & $4.71 \pm 3.61$ \\
\hline Mount Lebanon & $20.84 \pm 11.68$ & $5.14 \pm 3.55$ \\
\hline North & $17.95 \pm 10.28$ & $4.00 \pm 3.58$ \\
\hline South & $22.02 \pm 10.08$ & $5.26 \pm 3.02$ \\
\hline Beqaa & $23.17 \pm 14.49$ & $6.46 \pm 2.87$ \\
\hline$p$ & 0.022 & $<0.001$ \\
\hline Cumulative cigarette smoking & Rho $=0.567^{\mathrm{a}}$ & Rho $=0.503^{\mathrm{a}}$ \\
\hline Age & Rho $=-0.029$ & Rho $=-0.011$ \\
\hline House crowding index & Rho $=0.098$ & Rho $=0.043$ \\
\hline
\end{tabular}

Rho Spearman correlation coefficient

${ }^{\mathrm{a}} p<0.001$; numbers in italics indicate significant $p$ values

significantly associated with higher nicotine dependence, while cumulative cigarette smoking was significantly associated with higher nicotine dependence and symptoms. Also, females had lower nicotine symptoms than males.

\section{ACDS validation}

In our study, the ACDS items converged over a solution of three factors (factor $1=$ smoking patterns; factor $2=$ positive and negative reinforcements; factor $3=$ smoking cessation), explaining a total of $56.18 \%$ of the variance and leading to the internal consistency of Cronbach's $\alpha=0.875$. The YACD scale showed four factors: nicotine dependence (with an excellent Cronbach's alpha of 0.809), craving intensity (Cronbach's alpha 0.636), positive reinforcement (with good reliability, Cronbach's alpha of 0.638), and negative reinforcement (Cronbach's alpha 0.587) (Salameh et al. 2013). The development of the "Self-Administered Questionnaire to Measure Dependence on Cigarettes: The Cigarette Dependence Scale" and its short version (Etter et al. 2003) resulted in a 12-item scale labeled the Cigarette Dependence Scale (CDS-12) and a short 5-item version (CDS-5). Except for tolerance, CDS-12 covers the main components of DSMIV and ICD-10 definitions of dependence: compulsion, withdrawal symptoms, loss of control, time allocation, neglect of other activities, and persistence despite harm. CDS- 5 has similar measurement properties but less comprehensive content. Both scales had a high test-retest reliability $(r \geq 0.83)$ and a high internal consistency (Cronbach's alpha $\geq 0.84$ ). Thus, the validated version of the ACDS is a reliable tool to screen for cigarette dependence among Lebanese adolescents.

Our results showed a higher nicotine dependence (and higher smoking patterns) in participants with deceased/ divorced parents vs. living together. A recent Lebanese study could detect an association between parental divorce and higher cigarette and waterpipe addiction in adolescents (Jabbour et al. 2020). Research has also revealed that adolescents from divorced or separated families are more likely to use tobacco than those living with both parents (Mak et al. 2010), probably because parental divorce is negatively associated with social well-being among children and adolescents and positively related to risky health behaviors and delinquency (Sillekens and
Table 4 Multivariable analysis

\begin{tabular}{lcccc}
\hline Variable & UB & $S B$ & $p$ & $95 \%$ CI \\
Model 1 taking the ACDS score as the dependent variable & & & \\
Deceased parent vs living together & & & \\
a & 8.54 & 0.26 & $<0.001$ & $5.72-11.36$ \\
Divorced parents vs living together ${ }^{\mathrm{a}}$ & 4.26 & 0.12 & 0.007 & $1.19-7.32$ \\
Cumulative cigarette smoking & 0.29 & 0.53 & $<0.001$ & $0.24-0.34$ \\
Nagelkerke $R^{2}=43.8 \%$ & & & \\
Model 2 taking the HONC due to cigarettes score as the dependent variable & \\
Gender (females vs males ${ }^{\mathrm{a}}$ ) & -0.75 & -0.10 & 0.056 & $-1.51-0.20$ \\
Cumulative cigarette smoking & 0.05 & 0.32 & $<0.001$ & $0.03-0.07$ \\
Nagelkerke $R^{2}=14.1 \%$ & & & \\
\hline
\end{tabular}

$U B$ unstandardized beta, $S B$ standardized beta, $C I$ confidence interval

${ }^{\text {a }}$ Reference group; numbers in italics indicate significant $p$ values 
Table 5 MANCOVA multivariable analysis

\begin{tabular}{|c|c|c|c|c|}
\hline Variable & Beta & $p$ & $95 \% \mathrm{CI}$ & Partial Eta squared \\
\hline \multicolumn{5}{|c|}{ Model 1: Factor 1 (smoking patterns) as the dependent variable } \\
\hline Deceased parent vs living together ${ }^{\mathrm{a}}$ & 0.86 & $<0.001$ & $0.43-1.30$ & 0.115 \\
\hline \multicolumn{5}{|c|}{ Model 2: Factor 2 (positive and negative reinforcements) as the dependent variable } \\
\hline Deceased parent vs living together ${ }^{\mathrm{a}}$ & 1.25 & $<0.001$ & $0.80-1.70$ & 0.203 \\
\hline \multicolumn{5}{|c|}{ Model 3: Factor 3 (smoking cessation) as the dependent variable } \\
\hline Household crowding index & 2.10 & $<0.001$ & $1.74-2.47$ & 0.521 \\
\hline Deceased parent vs living together ${ }^{\mathrm{a}}$ & 1.23 & $<0.001$ & $0.93-1.53$ & 0.360 \\
\hline Divorced parent vs living together ${ }^{\mathrm{a}}$ & 1.94 & $<0.001$ & $0.93-2.95$ & 0.108 \\
\hline
\end{tabular}

${ }^{\text {a }}$ Reference group; variables entered in the analysis: age, gender, education level, household crowding index, and parents' status
Notten 2020). Furthermore, parental divorce, family conflict, and parents' death increase the likelihood of feelings of depression and anger among adolescents, which may increase risky behaviors, such as cigarette smoking (Lindstrom and Rosvall 2020). Indeed, family emotional stressors that often accompany marital discord, decreased time spent with parents, lack of parental monitoring, disruptive social changes, such as moving to a new home as a result of divorce or changing schools, may explain the increased risk for cigarette dependence among adolescents of divorced or deceased parents (Jabbour et al. 2020).

Our results also showed a positive correlation between the ACDS score and cumulative cigarette smoking. To the best of our knowledge, no previous studies have explored this association in adolescents. The YACD scale showed a correlation with carbon monoxide, the number of packs smoked per day, duration of smoking in years, and smoking cumulative dose (Salameh et al. 2013). Moreover, a study reported a doseresponse relationship with an increased risk for former smokers, a higher number of cigarettes smoked per day for current smokers, and a higher number of smoking years (Klungsoyr et al. 2006), indicating that cigarette dependence may go beyond physiological nicotine dependence (Centers for Disease Control and Prevention (US); National Center for Chronic Disease Prevention and Health Promotion (US); Office on Smoking and Health (US) 2010). Further studies are necessary to reveal the presence of different profiles of dependent smokers to design smoking cessation programs accordingly. More information is also needed on the array of genes involved in the metabolic processes and the toxicokinetic variation of nicotine and other tobacco substrates for a better appreciation of how these differences influence susceptibility to becoming nicotine or cigarette dependent.

Another result was a higher HONC score for a higher cumulative cigarette smoking, in line with a previous study (Soteriades et al. 2020), explained by the fact that nicotine activates the brain reward pathways and also leads to an increase in dopamine, which reinforces rewarding behaviors
(National Institute on Drug Abuse Cigarettes and Other Tobacco Products DrugFacts n.d.)

Our results revealed that a higher household crowding index (reflecting a lower socioeconomic status) was significantly correlated with more smoking cessation. Findings regarding this factor are contradictory. Research suggests that smoking cessation treatments may be less effective among individuals with lower SES (Hiscock et al. 2015). These effects are likely accentuated by higher exposure to advertising of tobacco products in disadvantaged neighborhoods (Linetzky et al. 2012). Thus, the environment of the school may impact smoking behaviors and cessation of students through several mechanisms, including exposure to tobacco advertising (Barreto et al. 2012), availability of tobacco products, and the development of social norms that facilitate or detract from youth smoking (Lovato et al. 2010). However, another study showed that the desire to quit smoking might be more frequent in more deprived schools (Linetzky et al. 2012). Our results may be linked to the inability of adolescents to purchase cigarettes, given their high price in Lebanon following the economic crisis. This finding unveils the need to increase access to interventions aimed at facilitating quitting (such as cognitive therapies and motivational interviewing) in different types of schools (public and private, with lower and higher SES).

Finally, having a deceased parent or divorced parents compared was living together was significantly associated with more smoking cessation. To the best of our knowledge, no previous studies have investigated the association between these two variables. However, parental divorce or parents' death is considered a stressful situation (Schaan et al. 2019). Previous studies suggest that individuals with higher perceived stress scores and more negative life events are less likely to quit smoking (Lawless et al. 2015); adolescents most often considered smoking as a coping strategy helping them to relax (McGee et al. 2013). On the other hand, another study (Siqueira et al. 2001) revealed that cognitive coping was the only coping method that showed a significant difference 
between smokers and quitters. More particularly, this coping method possibly helps Lebanese adolescents attempting to quit smoking to reframe stressful events or tempting situations; the cognitive strategy reduces their need to reach for a cigarette to cope with stressors. The scope of this approach includes thinking about the health benefits of not smoking, evaluating the social benefits of quitting (e.g., pleasing a parent or loved one), and using distraction strategies, such as thinking about pleasant alternative activities or a simple delay in smoking cigarettes.

\section{Limitations}

The limitations of this study might reside in the fact that it only explored factors at one point in time, not over a duration of time. A social desirability bias is also possible due to the fear of respondents to give honest answers; however, the anonymity of the questionnaire might have reduced this limitation. An information bias might have occurred during the data collection process since it was on a self-report survey. A selection bias is present because of the snowball technique used to collect the surveys. A residual confounding bias is also possible since not all factors associated with smoking were considered in the current questionnaire.

\section{Conclusion}

This study could validate a new tool to evaluate nicotine dependence among Lebanese adolescents, the Adolescent Cigarette Dependence Scale (ACDS). This version adapted from the YACD scale is reliable and valuable for use in clinical research. This study was also able to demonstrate the positive association between cumulative smoking and cigarette dependence. Public health strategies are essential to try decreasing the tobacco burden as much as possible; this includes implementation of laws in Lebanon related to banning cigarette smoking, increasing tobacco taxation, raising awareness about the bad consequences of cigarette smoking on one's health, and promoting healthy lifestyle among adolescents. However, further studies are necessary to improve this instrument, adding new questions useful for the diagnosis and evaluation of cigarette dependence.

Acknowledgements The authors would like to thank all students who helped them in the data collection.

Author contribution PS and SH conceived and designed the survey. SH and PS were involved in the statistical analysis and data interpretation. SH and $\mathrm{SO}$ wrote the manuscript. AK helped with the writing. MA involved the data collection. HS edited the paper. All authors read the manuscript, critically revised it for intellectual content, and approved the final version.
Data availability The authors do not have the right to share any data information as per their institutions' policies.

\section{Declarations}

Ethics approval and consent to participate The Psychiatric Hospital of the Cross Ethics and Research Committee waived the need for the approval of the study (HPC-031-2020). The purpose and requirements of the study were explained to each participant, and filling the form and submitting it online consented that the person approved to participate in the study.

Consent for publication Not applicable.

Competing interests The authors declare that they have no competing interests.

\section{References}

Bahelah R, DiFranza JR, Fouad FM, Ward KD, Eissenberg T, Maziak W (2016) Early symptoms of nicotine dependence among adolescent waterpipe smokers. Tob Control 25:e127-e134

Barreto SM, Giatti L, Casado L, de Moura L, Crespo C, Malta D (2012) Contextual factors associated with smoking among Brazilian adolescents. J Epidemiol Community Health 66:723-729

Bejjani N, El Bcheraoui C, Adib SM (2012) The social context of tobacco products use among adolescents in Lebanon (MedSPAD-Lebanon). J Epidemiol Glob Health 2:15-22

Centers for Disease Control and Prevention (US); National Center for Chronic Disease Prevention and Health Promotion (US); Office on Smoking and Health (US) (2010): How tobacco smoke causes disease: the biology and behavioral basis for smoking-attributable disease: a report of the surgeon general. Atlanta (GA): Centers for Disease Control and Prevention (US); 2010. 4, Nicotine Addiction: Past and Present. Available from: https://www.ncbi. nlm.nih.gov/books/NBK53018/. Accessed 29 Sept 2020

Clark DB, Wood DS, Martin CS, Cornelius JR, Lynch KG, Shiffman S (2005) Multidimensional assessment of nicotine dependence in adolescents. Drug Alcohol Depend 77:235-242

Comrey AL, Lee HB (2013): A first course in factor analysis. Psychology press

DiFranza JR, Rigotti NA, McNeill AD, Ockene JK, Savageau JA, St Cyr D, Coleman M (2000) Initial symptoms of nicotine dependence in adolescents. Tob Control 9:313-319

DiFranza JR, Savageau JA, Fletcher K, Ockene JK, Rigotti NA, McNeill AD, Coleman M, Wood C (2002) Measuring the loss of autonomy over nicotine use in adolescents: the DANDY (Development and Assessment of Nicotine Dependence in Youths) study. Arch Pediatr Adolesc Med 156:397-403

DiFranza JR, Savageau JA, Fletcher K, O'Loughlin J, Pbert L, Ockene JK, McNeill AD, Hazelton J, Friedman K, Dussault G, Wood C, Wellman RJ (2007) Symptoms of tobacco dependence after brief intermittent use: the Development and Assessment of Nicotine Dependence in Youth-2 study. Arch Pediatr Adolesc Med 161: 704-710

Dir AL, Bell RL, Adams ZW, Hulvershorn LA (2017) Gender differences in risk factors for adolescent binge drinking and implications for intervention and prevention. Front Psychiatry 8:289

Etter JF, Le Houezec J, Perneger TV (2003) A self-administered questionnaire to measure dependence on cigarettes: the cigarette dependence scale. Neuropsychopharmacology 28:359-370 
Fagerstrom KO (1978) Measuring degree of physical dependence to tobacco smoking with reference to individualization of treatment. Addict Behav 3:235-241

Griesler PC, Hu MC, Kandel DB (2016) Nicotine dependence in adolescence and physical health symptoms in early adulthood. Nicotine Tob Res 18:950-958

Hammerslag LR, Gulley JM (2016) Sex differences in behavior and neural development and their role in adolescent vulnerability to substance use. Behav Brain Res 298:15-26

Heatherton TF, Kozlowski LT, Frecker RC, Fagerstrom KO (1991) The Fagerstrom test for nicotine dependence: a revision of the Fagerstrom Tolerance Questionnaire. Br J Addict 86:1119-1127

Hiscock R, Dobbie F, Bauld L (2015) Smoking cessation and socioeconomic status: an update of existing evidence from a National Evaluation of English Stop Smoking Services. Biomed Res Int 2015:274056

Jabbour N, Abi Rached V, Haddad C, Salameh P, Sacre H, Hallit R, Soufia M, Obeid S, Hallit S (2020) Association between parental separation and addictions in adolescents: results of a National Lebanese Study. BMC Public Health 20:965

Klungsoyr O, Nygard JF, Sorensen T, Sandanger I (2006) Cigarette smoking and incidence of first depressive episode: an 11-year, population-based follow-up study. Am J Epidemiol 163:421-432

Lawless MH, Harrison KA, Grandits GA, Eberly LE, Allen SS (2015) Perceived stress and smoking-related behaviors and symptomatology in male and female smokers. Addict Behav 51:80-83

Lindstrom M, Rosvall M (2020) Parental separation/divorce in childhood and tobacco smoking in adulthood: A population-based study. Scand J Public Health 48:657-666

Linetzky B, Mejia R, Ferrante D, De Maio FG, Diez Roux AV (2012) Socioeconomic status and tobacco consumption among adolescents: a multilevel analysis of Argentina's Global Youth Tobacco Survey. Nicotine Tob Res 14:1092-1099

Lovato CY, Zeisser C, Campbell HS, Watts AW, Halpin P, Thompson M, Eyles J, Adlaf E, Brown KS (2010) Adolescent smoking: effect of school and community characteristics. Am J Prev Med 39:507514

Mak KK, Ho SY, Thomas GN, Schooling CM, McGhee SM, Lam TH (2010) Family structure, parent-child conversation time and substance use among Chinese adolescents. BMC Public Health 10:503
McGee R, Williams S, Nada-Raja S, Olsson CA (2013) Tobacco smoking in adolescence predicts maladaptive coping styles in adulthood. Nicotine Tob Res 15:1971-1977

National Institute on Drug Abuse Cigarettes and Other Tobacco Products DrugFacts. Available from: https://www.drugabuse.gov/ publications/drugfacts/cigarettes-other-tobacco-products

Salameh P, Jomaa L, Farhat G, Zeghondi H, Gerges N, Issa C, Sabbagh MT, Chaaya M, Barbour B, Waked M, Salame J, Saadallah-Zeidan N, Baldi I, Lebanese National Conference for Health in Universities study group (2013) The Young Adults' Cigarette Dependence (YACD) score: an improved tool for cigarette dependence assessment in university students. Addict Behav 38:2174-2179

Schaan VK, Schulz A, Schachinger H, Vogele C (2019) Parental divorce is associated with an increased risk to develop mental disorders in women. J Affect Disord 257:91-99

Shiffman S, Waters A, Hickcox M (2004) The nicotine dependence syndrome scale: a multidimensional measure of nicotine dependence. Nicotine Tob Res 6:327-348

Sillekens S, Notten N (2020) Parental divorce and externalizing problem behavior in adulthood. A study on lasting individual, family and peer risk factors for externalizing problem behavior when experiencing a parental divorce. Deviant Behav 41:1-16

Siqueira LM, Rolnitzky LM, Rickert VI (2001) Smoking cessation in adolescents: the role of nicotine dependence, stress, and coping methods. Arch Pediatr Adolesc Med 155:489-495

Soteriades S, Basagiannis A, Soteriades ES, Barbouni A, Mouchtouri V, Rachiotis G, Hadjichristodoulou C (2020) Nicotine dependence and loss of autonomy among Greek adolescent smokers: a countrywide cross-sectional study. Int J Environ Res Public Health 17

World Health Organization Behaviours affecting current and future health - tobacco use (2020). Available from: https://apps.who.int/ adolescent/second-decade/section4/page8/Tobacco-use.html [Accessed on October 1, 2020].

Publisher's Note Springer Nature remains neutral with regard to jurisdictional claims in published maps and institutional affiliations. 